\title{
Constructions and Some Search Results of Ternary LRCs with
} $d=6^{*}$

\author{
Youliang ZHENG ${ }^{\dagger a)}$, Ruihu $\mathbf{L I}^{\dagger \mathrm{b})}$, Jingjie $\mathbf{L V}^{\dagger c)}$, Nonmembers, and Qiang FU ${ }^{\dagger \mathrm{d})}$, Member
}

\begin{abstract}
SUMMARY Locally repairable codes (LRCs) are a type of new erasure codes designed for modern distributed storage systems (DSSs). In order to obtain ternary LRCs of distance 6, firstly, we propose constructions with disjoint repair groups and construct several families of LRCs with $1 \leq r \leq$ 6 , where codes with $3 \leq r \leq 6$ are obtained through a search algorithm. Then, we propose a new method to extend the length of codes without changing the distance. By employing the methods such as expansion and deletion, we obtain more LRCs from a known LRC. The resulting LRCs are optimal or near optimal in terms of the Cadambe-Mazumdar (C-M) bound. key words: locally repairable codes, parity-check matrix, disjoint repair groups
\end{abstract}

\section{Introduction}

The coming era of big data brings great pressure to modern distributed storage systems in terms of efficiency and reliability. The traditional triple replication scheme is no longer applicable due to its low efficiency [1], [2]. Therefore, locally repairable codes that can effectively solve such problems have emerged in recent years. When a storage node fails to function temporally or permanently, it can be recovered by accessing at most $r$ other nodes. The number $r$ is called the locality of the node. An LRC with locality $r$ means that all nodes have locality at most $r$.

Definition 1: An $[n, k, d ; r]_{q}$ LRC is a linear code of length $n$, dimension $k$, distance $d$ and locality $r$ over the finite field $\mathbb{F}_{q}$. [3]:

Gopalan et al. has proposed an upper bound of LRCs

$$
d \leq n-k-\left\lceil\frac{k}{r}\right\rceil+2,
$$

which is also called the Singleton-like bound since it reduces to the classical Singleton bound when $r=k$. Ternary LRCs reaching the Singleton-like bound has been given in [4]. The Singleton-like bound is not tight for codes over small finite fields. Cadambe and Mazumdar proposed a

\footnotetext{
Manuscript received June 28, 2020.

Manuscript publicized September 1, 2020

The authors are with the Department of Basic Sciences, Air Force Engineering University, Xi' an, P.R. China.

${ }^{*}$ This work is supported by National Natural Science Foundation of China (Nos.11801564,11901579) and the Graduate Scientific Research Foundation of Department of Basic Sciences.

a)E-mail: zxy951783247@163.com

b)E-mail: 1lzsy110@163.com

c)E-mail: juxianljj@163.com

d) E-mail: fuqiangkgd@163.com

DOI: $10.1587 /$ transfun.2020EAL2070
}

field-dependent bound as follows [5]:

An $[n, k, d ; r]_{q}$ LRC satisfies:

$$
k \leq \min _{t \in Z^{+}}\left\{t r+k_{o p t}^{q}(n-t(r+1), d)\right\},
$$

where $Z^{+}$denotes positive integer and $k_{o p t}^{q}(n, d)$ is the largest possible dimension of a code with length $n$, distance $d$, and alphabet size $q$. The bound (2) is called the CadambeMazumdar (C-M) bound. We use $[n, k, 6 ; r]$ to denote ternary LRCs with locality $r$ in this paper. An LRC reaching the $\mathrm{C}-\mathrm{M}$ bound is said to be optimal. If the dimension of LRCs are one less than the C-M bound, we call these LRCs almost optimal.

LRC with small field size is easier to be implemented on hardware, so it has been widely studied by scholars. Gopraju et al. [6] analyzed binary cyclic LRCs. Ernvall et al. proposed LRCs over a small alphabet in [7]. Yang et al. studied the locality of low dimensional ternary optimal codes in [7]-[9]. References [10]-[12] utilized $t$-spreads to construct binary LRCs of 6 with disjoint repair groups. Hao et al. [13] proposed a class of LRCs with $q \geq r-1, q=2^{n-k}$ and $d=4$ and found four classes of binary LRCs, which reach the Singleton-like bound. Chen et al. [14] proposed improved bounds and constructed some LRCs with distance 5 and 6. Calderbank and Fishburn studied maximal threeindependent subsets over $\mathbb{F}_{3}^{n}$ in [15].

In this paper, we propose a construction for ternary LRCs of distance 6 with disjoint repair groups inspired by Ref. [11]. Construction conditions for different $r$ are classified and discussed, then we construct several families of LRCs with $1 \leq r \leq 6$. To obtain more LRCs, some methods such as deleting and expanding the parity-check matrices, are also discussed.

An outline of this paper is as follows. In Sect. 2, we introduce some mathematical notations and definitions. Section 3 provides the construction of LRCs with disjoint repair groups, hence, we construct some optimal or almost optimal ternary LRCs. In Sect. 4 we propose a method of expanding the code length $n$ without changing distance and dimension of parity-check matrix. Section 5 concludes the paper.

\section{Preliminaries}

First we give some mathematical notations and definitions which will be used later:

- Let $\mathbf{v}=\left(v_{1}, v_{2}, \cdots, v_{n}\right)$ and $v_{i}$ be the $i$-th coordinate of $\mathbf{v}$. The support of $\mathbf{v}$ is denoted as $\operatorname{supp}(\mathbf{v})=\{i$ 
$\left.\mid v_{i} \neq 0\right\}$ and the Hamming weight of $\mathbf{v}$ is denoted as $\operatorname{wt}(\mathbf{v})=|\operatorname{supp}(\mathbf{v})|$.

- Let $I_{n}$ be the $n \times n$ identity matrix, and $\mathbb{F}_{3}=\{0,1,2\}$. $\mathbf{0}_{n}, \mathbf{1}_{n}$ and $\mathbf{2}_{n}$ denote the all-zero row vector, all-one row vector and all-two row vector of length $n$.

- Let $[i]=\{1,2, \cdots, i\}$ and $[a, b]=\{a, a+1, \cdots, b \mid a \leq$ $b\}, i, a$ and $b$ are three positive integers.

- For a linear code with an $(n-k) \times n$ parity-check matrix $H$, denote the $i$-th row of $H$ as $\mathbf{h}_{i}(i \in[n-k])$.

- Let $\otimes$ denote tensor product, and $\mathbb{F}_{q}^{m}$ denotes all of $m$ dimensional vector spaces in finite field $\mathbb{F}_{q}$.

Definition 2: ([11]) We say an $[n, k, d ; r]$ LRC has disjoint repair groups if there exists a set of local check $\mathbf{h}_{1}, \mathbf{h}_{2}, \cdots, \mathbf{h}_{l} \in H$ such that $\cup_{i=1}^{l} \operatorname{supp}\left(\mathbf{h}_{i}\right)=[n], w t\left(\mathbf{h}_{i}\right)=$ $r+1$ and $\operatorname{supp}\left(\mathbf{h}_{i}\right) \cap \operatorname{supp}\left(\mathbf{h}_{j}\right)=\emptyset$ for $1 \leq i \neq j \leq l$.

Definition 3: A generator matrix of 2-dimensional simplex code is

$$
S_{2}=\left(\begin{array}{l}
1011 \\
0112
\end{array}\right)
$$

a generator matrix of $k$-dimensional simplex code can be constructed through recursion formula

$$
S_{k}=\left(\begin{array}{cccc}
S_{k-1} & \mathbf{0}_{k-1}^{\top} & S_{k-1} & S_{k-1} \\
\mathbf{0}_{n_{k-1}} & 1 & \mathbf{1}_{n_{k-1}} & 2_{n_{k-1}}
\end{array}\right),
$$

where $n_{k-1}$ is the number of columns in $S_{k-1}$. It is easy to know that $S_{k}$ has $\frac{3^{k}-1}{2}$ columns.

Definition 4: ([14], [16]) Denote $\mathcal{G}_{q}(m, s)$ as all $s$ dimensional subspaces of $\mathbb{F}_{q}^{m}$. A subset $\mathcal{S} \subset \mathcal{G}_{q}(m, s)$ is called a sunflower if any two distinct element in $\mathcal{S}$ intersect in the same $t$-subspace $\mathrm{Z}$. Moreover, the $t$-subspace $\mathrm{Z}$ is called the center of $\mathcal{S}$ denoted by $\operatorname{Cen}(\mathcal{S})$.

\section{Construction of LRCs with Disjoint Repair Groups}

In this section, we will construct ternary LRCs of distance 6 with disjoint repair groups. Corresponding construct conditions are given for $r \in$ [2]. If $r \geq 3$, we first give uniform constraints, then algorithm 1 is proposed to construct LRCs, conveniently.

Let $C$ be an $[n, k, 6 ; r]$ LRC code. We assume that the parity-check matrix $H$ of $C$ consists of two parts:

$$
H=\left(\begin{array}{c}
H_{L} \\
H_{G}
\end{array}\right) .
$$

As the local check, the upper block $H_{L}$ is designed to guarantee the locality $r$ of the code $C$. Suppose that $l=\frac{n}{r+1}$, where $n$ is the code length of $C$. We generally design $H_{L}$ as $I_{l} \otimes \mathbf{1}_{r+1}$. If we regard $m$ as the number of rows of $H$ and let $u=m-l$, then $H_{G}$ can be represented as an $u \times n$ matrix. The function of $H_{G}$ is that determines the minimum distance of $C$. Moreover, the parity-check matrix $H$ can be represented as follows [10]-[12]:

$$
H=\left(\begin{array}{cccc}
\mathbf{1}_{r+1} & \mathbf{0}_{r+1} & \cdots & \mathbf{0}_{r+1} \\
\mathbf{0}_{r+1} & \mathbf{1}_{r+1} & \cdots & \mathbf{0}_{r+1} \\
\vdots & \vdots & \ddots & \vdots \\
\mathbf{0}_{r+1} & \mathbf{0}_{r+1} & \cdots & \mathbf{1}_{r+1} \\
H_{G}^{1} & H_{G}^{2} & \cdots & H_{G}^{l}
\end{array}\right),
$$

where $H_{G}^{i}(i \in[l])$ denotes the $i$-th $u \times(r+1)$ sub-matrix of $H_{G}$. Because each column of $H_{L}$ exists and only exists one non-zero element, it follows the conditions that $\cup_{i=1}^{l} \operatorname{supp}\left(\mathbf{h}_{i}\right)=[n], w t\left(\mathbf{h}_{i}\right)=r+1$ and $\operatorname{supp}\left(\mathbf{h}_{i}\right) \cap \operatorname{supp}\left(\mathbf{h}_{j}\right)=$ $\emptyset$ for $1 \leq i \neq j \leq l$. Hence, we partition $H$ into $l$ disjoint repair groups, which are denoted as $H_{1}, H_{2}, \cdots, H_{l}$.

Given a linear code with a parity-check matrix $H$, it is well known that its distance is 6 if and only if any 5 columns in $H$ are linearly independent. Since $H$ has been divided into $l$ disjoint repair groups, we can prove $d=6$ through proving any 5 columns picked from these groups are linearly independent, which will make it easier to classify and discuss. By the elementary row transformations of the matrix, $H_{G}^{i}$ can be further transformed into the following format:

$$
H_{G}^{i}=\left(\mathbf{0}_{u}^{T} G^{i}\right),
$$

where $G^{i}$ consists of the $r$ non-zero column vectors in the $H_{G}^{i}$, and called basic vectors set of the $H_{G}^{i}$.

\subsection{LRCs with $r \in[2]$}

The construction of parity-check matrix as (3) is used here. $N_{u_{r}}$ as the maximum number of disjoint repair groups for given $r$ and $u$. Our work includes some previous results. Reference [4] has given the constructions of $[8,2,6 ; 1]$, $[9,3,6 ; 2]$ and $[12,5,6 ; 2]$. In addition, a [39,22,6;2] LRC is proved to exist in [14].

Lemma 1: Let $r=1, u \geq 2$ and $N_{u_{1}}=\frac{3^{u}-1}{2}$. For $i \neq$ $j \leq\left[N_{u_{1}}\right], \lambda_{1}, \lambda_{2} \in$ [2], if $\alpha^{i}$ in $G^{i}$ and $\alpha^{j}$ in $G^{j}$ satisfy $\lambda_{1} \alpha^{i}+\lambda_{2} \alpha^{j} \neq 0$, then there are [2l,l-u,6;1] LRCs for $l \in\left[u+1, N_{u_{1}}\right]$,

Proof We use $(2,1)$ to denote picking two columns from a disjoint group and one column from the other group. Similarly, when picking any two or three columns in $H$, it is easy to know that they are linearly independent. When picking four columns in $H$, there are 3 cases $(1,1,1,1),(2,1,1)$ and $(2,2)$, respectively; when picking five columns in $H$, there are 3 cases $(1,1,1,1,1),(2,1,1,1)$ and $(2,2,1)$, respectively. Among these cases, $(2,2)$ is linearly independent according to $\lambda_{1} \alpha^{i}+\lambda_{2} \alpha^{j} \neq 0$, the linear independence of $(2,2,1)$ can be derived from case $(2,2)$, other cases are linearly independent obviously. Due to any 5 columns are linearly independent in $H$, the distance can be proved to be 6 . For the range of $l$, since the dimensions of LRCs have to be greater than or equal to $r$, so the minimum value of $l$ is $u+1$. And we find that the vectors of simplex code satisfy the inequality condition, as a result, $N_{u_{1}}=\frac{3^{u}-1}{2}$. 
Example 1: If $u=3, l=5$, there exists an almost optimal $[10,2,6 ; 1]$ LRC, whose parity-check matrices $H$ is

$$
H=\left(\begin{array}{l}
1100000000 \\
0011000000 \\
0000110000 \\
0000001100 \\
0000000011 \\
0000010101 \\
0001000100 \\
0100000001
\end{array}\right) .
$$

Lemma 2: Let $r=2, u \geq 3$ and $N_{u_{2}}=\frac{3^{u-1}-1}{2}$. If the following conditions are satisfied, then there are $[3 l, 2 l-u, 6 ; 2]$ LRCs for $l \in\left[\left\lceil\frac{u+2}{2}\right\rceil, N_{u_{2}}\right]$.

1. The column vectors among each $G^{i}\left(i \in\left[N_{u_{2}}\right]\right)$ are linearly independent;

2. For $i \neq j \in\left[N_{u_{2}}\right], \lambda_{1}, \lambda_{2} \in \mathbb{F}_{3}, \lambda_{2} \neq 0$, two different columns $\alpha_{a}^{i}, \alpha_{b}^{i}$ in $G^{i}$ and two different columns $\alpha_{x}^{j}, \alpha_{y}^{j}$ in $G^{j}$ :

(1) $\alpha_{a}^{i}+\lambda_{1} \alpha_{b}^{i} \neq \lambda_{2} \alpha_{x}^{j}$;

(2) $\alpha_{a}^{i}+\lambda_{2} \alpha_{b}^{i} \neq \alpha_{x}^{j}+2 \alpha_{y}^{j}$;

Proof The proof of $d=6$ is similar to the proof in [14] and will not be repeated here. For the minimum value of $l$, since $2 l-u$ has to be greater than 2 , so $l>\left\lceil\frac{u+2}{2}\right\rceil$. By looking at the inequalities of condition 2 , we can see that the space based on $\alpha_{a}^{i}, \alpha_{b}^{i}$, and the space based on $\alpha_{x}^{j}, \alpha_{y}^{j}$ only exist an intersect vector $\alpha_{a}^{i}+\alpha_{b}^{i}=\alpha_{x}^{j}+\alpha_{y}^{j}$. If we regard $\alpha_{a}^{i}+\alpha_{b}^{i}$ as $\operatorname{Cen}(\mathcal{S})$, then, $t=1$. For $\mathcal{G}_{q}(m, s)$, let $m=u$, $s=2$, a sunflower $\mathcal{S}$ in $\mathcal{G}_{q}(u, 2)$ is the set of lines passing through a given point in the finite projective plane $P G(u, q)$. Therefore, the size of the sunflower $\mathcal{S}$ in $\mathcal{G}_{q}(u, 2)$ is $\frac{q^{u-1}-1}{q-1}$. When $q=3$, the size is $\frac{3^{u-1}-1}{2}$. Hence, the range of $l$ is $\left[\left\lceil\frac{u+2}{2}\right\rceil, N_{u_{2}}\right]$.

Example 2: If $u=4, l=4$, there exists an almost optimal $[12,4,6 ; 2] \mathrm{LRC}$, whose a parity-check matrix $H$ is

$$
H=\left(\begin{array}{l}
111000000000 \\
000111000000 \\
000000111000 \\
000000000111 \\
001001001010 \\
001001010001 \\
001010001001 \\
010001001001
\end{array}\right) .
$$

\subsection{LRCs with $r \geq 3$}

In this section, we give uniform conditions for constructing LRC with $r \geq 3$. Following an proposed search algorithm, some kinds of optimal and almost optimal LRCs with $3 \leq$ $r \leq 6$ can be obtained. Here we assume $N_{u_{r}}$ as obtained maximum number of disjoint repair groups for given $r$ and $u$ by the search algorithm.

Theorem 1: Let $r \geq 3$ and $u \geq 4$. If the following conditions are satisfied, then there are $[l(r+1), l r-u, 6 ; r]$ LRCs for $l \in\left[\left\lceil\frac{u+r}{r}\right\rceil, N_{u_{r}}\right]$.

1. Any $k$ columns in an disjoint repair group are linearly independent;

when $r=3, k=4$

when $r \geq 4, k=5$

2. For $i \neq j \in\left[N_{u_{r}}\right], \lambda_{1}, \lambda_{2} \in \mathbb{F}_{3}, \lambda_{2} \neq 0$, any three different columns like $\alpha_{a}^{i}, \alpha_{b}^{i}, \alpha_{c}^{i}$ in $G^{i}$ and any two different columns like $\alpha_{x}^{j}, \alpha_{y}^{j}$ in $G^{j}$ :

(1) $\alpha_{a}^{i}+\lambda_{1} \alpha_{b}^{i} \neq \lambda_{2} \alpha_{x}^{j}$;

(2) $\alpha_{a}^{i}+\lambda_{2} \alpha_{b}^{i} \neq \alpha_{x}^{j}+2 \alpha_{y}^{j}$;

(3) $\alpha_{a}^{i}+\alpha_{b}^{i}+\alpha_{c}^{i} \neq \lambda_{2} \alpha_{x}^{j}$;

(4) $\alpha_{a}^{i}+\alpha_{b}^{i}+\alpha_{c}^{i} \neq \alpha_{x}^{j}+2 \alpha_{y}^{j}$;

Proof When picking any two or three columns in $H$, it is easy to know that they are linearly independent according to condition 1 . When picking four columns in $H$, there are 5 cases, $(4),(3,1),(1,1,1,1),(2,1,1)$, and $(2,2)$, respectively; The first four are linearly independent obviously, the fifth case is linearly independent according to $\alpha_{a}^{i}+\lambda_{1} \alpha_{b}^{i} \neq \lambda_{2} \alpha_{x}^{j}$ and $\alpha_{a}^{i}+\lambda_{2} \alpha_{b}^{i} \neq \alpha_{x}^{j}+2 \alpha_{y}^{j}$. When picking five columns in $H$, there are 6 cases, $(1,1,1,1,1),(2,1,1,1),(2,2,1),(3,1,1)$, $(4,1)$ and $(3,2)$, respectively. The first five possibilities can be derived from the previous proof, and the sixth case is linearly independent according to $\alpha_{a}^{i}+\alpha_{b}^{i}+\alpha_{c}^{i} \neq \lambda_{2} \alpha_{x}^{j}$ and $\alpha_{a}^{i}+\alpha_{b}^{i}+\alpha_{c}^{i} \neq \alpha_{x}^{j}+2 \alpha_{y}^{j}$, so we can prove $d=6$. Since the dimension of LRC needs to be greater than the locality, it is easy to know that the value range of $l$ is $\left[\left\lceil\frac{u+r}{r}\right\rceil, N_{u_{r}}\right]$.

As $r$ and $u$ increase, one can see that it is difficult to determine $N_{u_{r}}$ and get the $r$ non-zero columns of $H_{G}^{i}$. So, a search algorithm is proposed to solve this problem. Here are some related concepts:

- $V_{u}$ represents a set, which includes $u$-dimensional space vectors without zero vector.

- $G_{m}^{i}$ : the vectors generated by $G^{i}$, mainly includes the following three parts: $G^{i}, 2 G^{i}$, the sum of any one basis vector and 2 times of another basis vector in $G^{i}$.

- $G_{M}$ : all vectors of each $G_{m}^{i}(i \in[l])$.

- $G_{n}^{i}$ : the vectors generated by $G^{i}$, mainly includes the sum of any two or three basis vectors and the vectors that are twice of the sum.

- $G_{N}$ : all vectors of each $G_{n}^{i}(i \in[l])$.

- $G_{N}^{*}$ : the set of vectors $V_{u} \backslash G_{M}$.

- $G_{M}^{*}$ : the set of vectors $V_{u} \backslash\left(G_{N} \cup G_{M}\right)$.

- $r_{u}$ : a set of $u$-dimensional column vectors that contain $r$ columns.

Corollary 1: For an $[l(r+1), l r-u, 6 ; r]$ LRC, assume an $r_{u}$ as $G^{j}(j=l+1)$, only if the following conditions are met: 1. Any $k$ columns in $H^{j}$ are linearly independent; when $r=3, k=4$; when $r \geq 4, k=5$;

2. $G_{m}^{j}$ is disjoint with $G_{m}^{i}$ and $G_{n}^{i}(i \in[l])$;

3. $G_{n}^{j}$ is joint with $G_{n}^{i}$ and disjoint with $G_{m}^{i}(i \in[l])$; then the $r_{u}$ can be regarded as a new basic vectors group, and an $[(l+1)(r+1), r(l+1)-u, 6 ; r]$ LRC can be constructed 


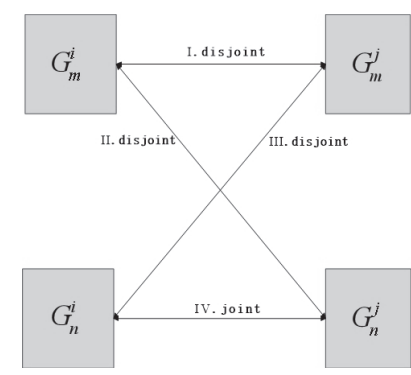

Fig. 1 Joint and disjoint relationship diagram.

by adding $G^{j}$ to $H_{G}^{j}$.

Proof According to the conditions of Corollary 1, we give a relationship diagram, as shown in Fig. 1. For $\lambda_{1} \in \mathbb{F}_{3}, \lambda_{2} \in$ [2]. Relationship I can derive 3 inequations: (1) $\alpha_{a}^{i} \neq \lambda_{2} \alpha_{x}^{j}$; (2) $\alpha_{a}^{i}+2 \alpha_{b}^{i} \neq \lambda_{2} \alpha_{x}^{j}$; (3) $\alpha_{a}^{i}+2 \alpha_{b}^{i} \neq \alpha_{x}^{j}+2 \alpha_{y}^{j}$. Relationships II and III can derive 4 inequations: (1) $\alpha_{a}^{i}+\alpha_{b}^{i} \neq \lambda_{2} \alpha_{x}^{j}$; (2) $\alpha_{a}^{i}+\alpha_{b}^{i} \neq \alpha_{x}^{j}+2 \alpha_{y}^{j}$; (3) $\alpha_{a}^{i}+\alpha_{b}^{i}+\alpha_{c}^{i} \neq \lambda_{2} \alpha_{x}^{j}$; (4) $\alpha_{a}^{i}+\alpha_{b}^{i}+\alpha_{c}^{i} \neq$ $\alpha_{x}^{j}+2 \alpha_{y}^{j}$. Relationship IV is an additional remark. So, the conditions of Corollary 1 are similar to the conditions of Theorem 1.

According to the definitions of $G_{M}^{*}$ and $G_{N}^{*}$, conditions 2 in Corollary 1 imply $G_{m}^{j} \subseteq G_{M}^{*}$ and conditions 3 in Corollary 1 imply $G_{n}^{j} \subseteq G_{N}^{*}$. Therefore, we can construct LRCs with $r \geq 3$ using Algorithm 1 as follows.

1. Set $r$ and $u(r \geq 3, u \geq 4)$;

2. Let $l=1, i=1, G_{M}^{*}=V_{u}, G_{N}^{*}=V_{u}$;

3. While:

4. Choose $r_{u} \in G_{M}^{*}$ as $G^{l}$ and add to $H_{G}^{l}$; $G^{l}$;

5. $G_{m}^{l}=\left\{\lambda g_{a}, \lambda\left(g_{a}+2 g_{b}\right) \mid a \neq b, \lambda \in[2], g_{a}, g_{b} \in\right.$

6. $G_{n}^{l}=\left\{\lambda\left(g_{a}+g_{b}\right), \lambda\left(g_{a}+g_{b}+g_{c}\right) \mid a \neq b \neq\right.$ $\left.c, \lambda \in[2], g_{a}, g_{b}, g_{c} \in G^{l}\right\}$;

7. If dent;

(1)Any $k$ columns in $H_{G}^{l}$ are linearly indepen-

when $r==3, k=4$;

when $r \geq 4, k=5$;

(2) $G_{m}^{l} \subseteq G_{M}^{*}$;

(3) $G_{n}^{l} \subseteq G_{N}^{*}$,

8. $\quad$ Let $i=1$, update $G_{M}^{*}$ and $G_{N}^{*}$;

9. Else

Let $i=i+1$

10. If $i>1000$

11. Break

12. Let $l=l+1$

13. Let $N_{u_{r}}=l$

14. Output $H_{G}^{i}(i \in[l])$ to construct $H$.

This is a search algorithm named Algorithm 1. The algorithm mainly transforms the linear restrict conditions of condition 2 in theorem 1 into the judgment of the inclusion
Table 1 Some optimal and almost optimal LRCs for given locality $r$.

\begin{tabular}{ccccc}
\hline num & $r$ & $u$ & $N_{u_{r}}$ & {$[n, k, 6 ; r]$} \\
\hline 1 & 3 & 4 & 4 & {$[4 l, 3 l-4,6 ; 3], 3 \leq l \leq 4$} \\
2 & 3 & 5 & 11 & {$[4 l, 3 l-5,6 ; 3], 3 \leq l \leq 11$} \\
3 & 4 & 5 & 4 & {$[5 l, 4 l-5,6 ; 4], 3 \leq l \leq 4$} \\
4 & 4 & 6 & 12 & {$[5 l, 4 l-5,6 ; 4], 4 \leq l \leq 6$} \\
5 & 5 & 4 & 2 & {$[6 l, 5 l-4,6 ; 5], l=2([4])$} \\
6 & 5 & 5 & 3 & {$[6 l, 5 l-5,6 ; 5], 2 \leq l \leq 3$} \\
7 & 5 & 6 & 6 & {$[6 l, 5 l-6,6 ; 5], 4 \leq l \leq 6$} \\
8 & 6 & 6 & 4 & {$[7 l, 6 l-6,6 ; 6], 3 \leq l \leq 4$} \\
\hline
\end{tabular}

relation of the set, thus reducing the computation and the time complexity. The space complexity of the algorithm is mainly determined by the dimension of the vector space searched. Since the judgment conditions of the algorithm is based on the linear relationships between the vectors within 5 columns, when the values of $u$ and $r$ are increased, the algorithm will output more disjoint repair groups, and would terminate when there is no new $H_{G}^{l}$ that satisfies the judge conditions after 1000 cycles. Through Algorithm 1, we got some optimal and almost optimal LRCs with $r \in[3,6]$, see Table 1.

Below we give the specific parity-check matrices $H$ of the codes with the most disjoint repair groups in each class. The parity-check matrices $H$ of other codes in the same class can be obtained by deleting corresponding disjoint repair groups and redundant zero element rows from the $H$ we wrote.

1. When $r=3, u=4, l=4$, there is an $[16,8,6 ; 3]$ LRC, whose a parity-check matrix $H$ is

$$
H=\left(\begin{array}{l}
1111000000000000 \\
0000111100000000 \\
0000000011110000 \\
0000000000001111 \\
0120012001210000 \\
0202021100010020 \\
0202020200220002 \\
0211011200210200
\end{array}\right) .
$$

2. When $r=3, u=5, l=11$, there is an $[44,28,6 ; 3]$ $\mathrm{LRC}$, whose a parity-check matrix $H$ is

$H=$

11110000000000000000000000000000000000000000 00001111000000000000000000000000000000000000 00000000111100000000000000000000000000000000 00000000000011110000000000000000000000000000 00000000000000001111000000000000000000000000 00000000000000000000111100000000000000000000 00000000000000000000000011110000000000000000 00000000000000000000000000001111000000000000 00000000000000000000000000000000111100000000 00000000000000000000000000000000000011110000 00000000000000000000000000000000000000001111 01000001021001210022002102220200001000110112 00100002000000210202010102200001001102020122 00010001020100220000020100110110022002100022 00000100021201210012010002120101010002200211 00000010001102110222011201000111001200220001 
3. When $r=4, u=5, l=4$, there is an $[20,11,6 ; 4]$ LRC, whose a parity-check matrix $H$ is

$$
H=\left(\begin{array}{l}
11111000000000000000 \\
00000111110000000000 \\
00000000001111100000 \\
00000000000000011111 \\
00220021100200201022 \\
01110000200022101100 \\
00210010120121202102 \\
02112000120201002212 \\
02102011210222102121
\end{array}\right) .
$$

4. When $r=4, u=6, l=6$, there is an $[30,18,6 ; 4]$ LRC, whose a parity-check matrix $H$ is

$$
H=\left(\begin{array}{l}
111110000000000000000000000000 \\
000001111100000000000000000000 \\
000000000011111000000000000000 \\
000000000000000111110000000000 \\
000000000000000000001111100000 \\
000000000000000000000000011111 \\
022110002200001021010112002200 \\
002200101000011022110220000011 \\
021220010202120001110000201201 \\
022110122102120012000211000002 \\
011210022200222011200100101201 \\
000000121101200012120101202121
\end{array}\right) .
$$

5. When $r=5, u=4, l=2$, there is an $[12,6,6 ; 5]$ LRC, whose a parity-check matrix $H$ is

$$
H=\left(\begin{array}{l}
111111000000 \\
000000111111 \\
002121000210 \\
020220000222 \\
000201020112 \\
000111002022
\end{array}\right) .
$$

6. When $r=5, u=5, l=3$, there is an $[18,10,6 ; 5]$ LRC, whose a parity-check matrix $H$ is

$$
H=\left(\begin{array}{l}
111111000000000000 \\
000000111111000000 \\
000000000000111111 \\
002211021120010021 \\
001110010002012112 \\
010110001101021201 \\
012210000201011002 \\
001212000102011211
\end{array}\right) .
$$

7. When $r=5, u=6, l=6$, there is an $[36,24,6 ; 5]$ LRC, whose a parity-check matrix $H$ is

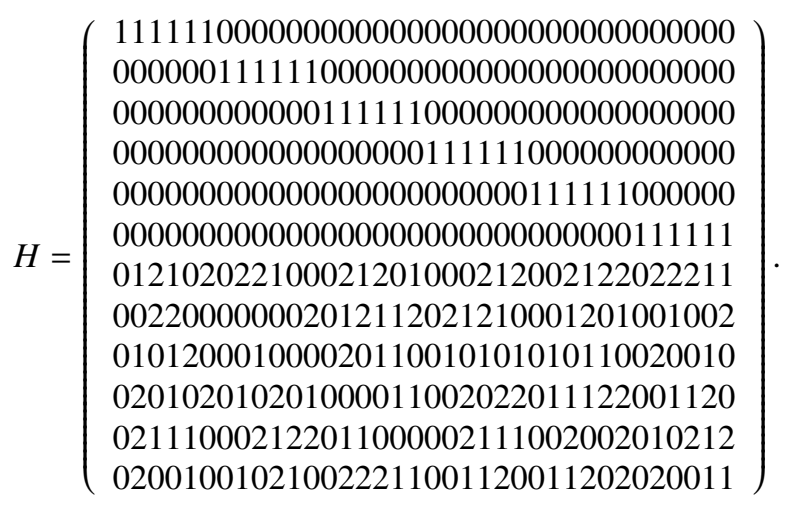

8. When $r=6, u=6, l=4$, there is an $[28,18,6 ; 6]$ LRC, whose a parity-check matrix $H$ is

$$
H=\left(\begin{array}{l}
1111111000000000000000000000 \\
0000000111111100000000000000 \\
0000000000000011111110000000 \\
0000000000000000000001111111 \\
0100012011212201211010002122 \\
0222020011122202000220222202 \\
0101201021221102221200022022 \\
0020001020120002121220120002 \\
0222200001000101010210101122 \\
0212020020201102012100121000
\end{array}\right) .
$$

\section{Expanding Code Length of LRCs}

For an $[n, k, 6 ; r] \mathrm{LRC}$ with a parity-check matrix $H$, there is an algorithm to expand the code length $n$ without changing the distance 6 and the dimension of parity-check matrix. The content of the Algorithm 2 is as follows.

1. Let $i=0, V_{(0)}=V_{m}, H_{(0)}=H$. //initialization.

2. While :

3. Set $S_{i}=\left\{a \mid a=\Sigma_{j=1}^{4} \lambda_{j} \mathbf{v}_{j}, \lambda_{j} \in[0,2], \mathbf{v}_{j} \in\right.$ $\left.H_{(i)}\right\}$. $\quad / /$ pick a $\operatorname{set}\left(S_{i}\right)$ of column vectors that are linear combinations of the column vectors within 4 columns in $H_{(i)}$.

4. Let $V_{(i+1)}=V_{(i)} \backslash S_{i}$. $\quad / /$ get a set of $V_{(i+1)}$, of which any one column and any 4 columns in $H_{(i)}$ are 5 linearly independent.

5. If $V_{(i+1)} \neq \emptyset$

6. Choose $\mathbf{v}, \mathbf{v} \in V_{(i+1)} \quad$ //Pick any a vector $\mathbf{v}$ from $V_{(i+1)}$.

7. Let $H_{(i+1)}=\left[H_{(i)}, \mathbf{v}\right]$.

8. $\quad i=i+1$.

9. Else

10. Output $H_{(i)}$.

11. Break.

If $H$ increases $i$ columns, we can get an $\left[n+i, k+i, 6 ; r^{*}\right]$ LRC with $r^{*} \in[r+1, r+i]$.

Example 3: For an optimal $[20,11,6 ; 4]$ LRC, by using the method of expanding code length from $V_{9}, n$ can be at most 
up to 24 . The codes with $n \in[21,24]$ are optimal. A paritycheck matrix of $[24,15,6 ; 7] \mathrm{LRC}$ is

$$
H=\left(\begin{array}{lll:l}
11111000000000000000 & 0111 \\
00000111110000000000 & 1011 \\
00000000001111100000 & 1202 \\
00000000000000011111 & 2120 \\
00220021100200201022 & 0111 \\
01110000200022101100 & 1021 \\
00210010120121202102 & 1021 \\
02112000120201002212 & 0001 \\
02102011210222102121 & 2001
\end{array}\right) .
$$

Using methods such as expansion and deletion, we can construct some optimal and almost optimal LRCs as follows:

An optimal $[13,6,6 ; 3]$ LRC can be constructed by expanding $[12,5,6 ; 2]$ LRC. An optimal $[11,4,6 ; 2]$ LRC is constructed by deleting $[12,5,6 ; 2]$.

When $r=3, u=4, l=4$, an optimal $[16,8,6 ; 3]$ LRC is obtained in Table 1 ; an almost optimal $[14,6,6 ; 3]$ LRC and an optimal $[15,7,6 ; 3]$ LRC can be constructed by deleting [16, 8, 6; 3]; optimal LRCs for $n \in[17,28]$ (expect $n=18,19)$ are constructed by expanding $[16,8,6 ; 3]$.

When $r=4, u=5, l=4$, we can construct an optimal $[20,11,6 ; 4]$ LRC in Table 1 ; almost optimal LRCs for $n \in[18,19]$ are constructed by deleting [20,11,6;4]; optimal LRCs for $n \in[21,24]$ are constructed by expanding $[20,11,6 ; 4]$.

\section{Conclusions}

In this paper, we proposed a construction for ternary LRCs based on disjoint repair groups with distance 6. All codes obtained from our construction are optimal or almost optimal according to the $\mathrm{C}-\mathrm{M}$ bound. In the future, we think that the properties of obtained LRCs have the possibility of further optimization, and we want to construct some optimal LRCs with other distances.

\section{References}

[1] H. Weatherspoon and J.D. Kubiatowicz, "Erasure coding vs. replication: A quantitative comparison," International Workshop on Peerto-Peer Systems, pp.328-337, 2002.
[2] A.G. Dimakis, P.B. Godfrey, and Y. Wu, "Network coding for distributed storage," IEEE Trans. Inf. Theory, vol.56, no.9, pp.45394551, 2010.

[3] P. Gopalan, C. Huang, H. Simitci, and S. Yekhanin, "On the locality of codeword symbols," IEEE Trans. Inf. Theory, vol.58, no.11, pp.6925-6934, 2012.

[4] J. Hao, S. Xia, and B. Chen, "On optimal ternary locally repairable codes," IEEE International Symposium on Information Theory, pp.171-175, 2017.

[5] V. Cadambe and A. Mazumdar, "An upper bound on the size of locally recoverable codes," IEEE International Symposium on Network Coding, pp.1-5, 2013.

[6] S. Gopraju and R. Calderbank, "Binary cyclic codes that are locally repairable," IEEE International Symposium on Information Theory, pp.676-680, 2014.

[7] T. Ernvall, T. Westerback, R. Freij-Hollanti, and C. Hollanti, "Constructions and properties of linear locally repairable codes," IEEE Trans. Inf. Theory, vol.62, no.3, pp.1129-1143, 2015.

[8] R. Yang, R. Li, L. Guo, Q. Fu, and Y. Rao, "Locality of some optimal ternary linear codes," Procedia Computer Science, vol.107, pp.164 169, 2016.

[9] L. Guo, Q. Fu, G. Chen, and R. Yang, "Locality of some optimal ternary codes," Proc. 3rd Annual International Conference on Information System and Artificial Intelligence, paper number 012077 , 2018

[10] M.Y. Nam and H.Y. Song, "Binary locally repairable codes with minimum distance at least six based on partial $t$-spreads," IEEE Commun. Lett., vol.21, no.8, pp.1683-1686, 2017.

[11] J. Ma and G. Ge, "Optimal binary linear locally repairable codes with disjoint repair groups," arXiv:1711.07138v1, 2017.

[12] C. Kim and J.S. No, "New constructions of binary LRCs with disjoint repair groups and locality 3 using existing LRCs," IEEE Commun. Lett., vol.23, no.3, pp.406-409, 2019.

[13] J. Hao, S. Xia, and B. Chen, "Some results on optimal locally repairable codes," IEEE International Symposium on Information Theory, pp.440-444, 2016.

[14] B. Chen, S. Xia, and J. Hao, "Improved bounds and optimal constructions of locally repairable codes with distance 5 and 6," IEEE International Symposium on Information Theory, pp.430-434, 2019.

[15] A.R. Calderbank and P.C. Fishburn, "Maximal three-independent subsets of $\{0,1,2\}^{n}$," Des. Codes Crypt., vol.4, pp.203-211, 1994

[16] T. Etzion and L. Storme, "Galois geometries and coding theory," Des. Codes Crypt., vol.78, pp.311-350, 2016. 